\title{
THE PRESERVATION OF BACTERIA BY DRYING IN PEPTONE PLUGS*
}

\author{
BY D. I. ANNEAR \\ Department of Pathology, University of Cambridge \\ (With Plates 15 and 16, and 4 Figures in the Text)

\section{INTRODUCTION}

In a previous paper (Annear, 1954) a new method for preserving bacteria was outlined. Briefly, the method consisted of dehydrating in vacuo the viscous product which resulted when a small volume of bacterial suspension was incorporated in a plug of sterile freeze-dried peptone.

The three purposes of the present paper are: (1) to describe the method in more detail; (2) to present some data on the preservation of some strains of the two genera Neisseria and Vibrio, which have hitherto been regarded as exceptionally sensitive to drying; and (3) to present some data on the relationship for several organisms between the growth phase and recoveries after drying.

The preparation, from freeze-dried peptone, of foams which may be useful for the preservation of micro-organisms is described in an appendix.

A general review of the preservation of bacteria by drying was recently made by Fry (1954).

\section{GENERAL METHODS}

Organisms

All but two of the strains dried are listed in Table 2. The two exceptions are a departmental (P.D.) strain of Staphylococcus aureus and a paracolon bacillus (N.C.T.C. 9793). This latter organism was kindly supplied by Dr R. M. Fry and is more familiarly known as $\mathrm{D} 201 \mathrm{H}$ by the numerous workers who have used it. Strain 32619 of Neisseria gonorrhoeae was a fresh isolate from Addenbrooke's Hospital when dried.

\section{Media}

Nutrient broth. This was a digest broth of ox-heart muscle and was prepared essentially by Hartley's method.

Nutrient agar. Nutrient broth with approximately $2 \%$ agar.

Blood-agar. Nutrient agar with 5-7\% oxalated horse blood.

Chocolate-agar. As for blood-agar but heated for about $10 \mathrm{~min}$. at $75^{\circ} \mathrm{C}$.

Suspending media. Suspending media for the inocula of the plugs were broth diluted with distilled water, aqueous glucose solutions, or diluted broth with various concentrations of glucose. The aqueous glucose solutions were 'sterilized'

* This paper is based on a thesis accepted in November 1955 for the $\mathrm{Ph} . \mathrm{D}$. degree of the University of Cambridge. 
by boiling for brief periods or by steaming. Where required, sterile broth was added to the glucose solutions, but not heated with them.

Diluent. Nutrient broth was used for dissolving the desiccates and also for diluting suspensions for the purposes of counting.

\section{Preparation of freeze-dried plugs}

Early efforts in this work were largely concerned with the production of a compact peptone plug which would absorb but not be too rapidly dissolved by the small volume of bacterial suspension which was later dropped on it. Suitable specifications were determined by trial and error. Later, substances other than peptone were investigated. The materials were sterilized either by heat or by filtration and dried by evaporation from the frozen state. The freeze-drying model used for this purpose was a prototype of the L 3 model made by Edwards High Vacuum Ltd. Its essential features were a refrigerated coil operating at $-40^{\circ} \mathrm{C}$. and a vacuum chamber with an operational pressure of $0 \cdot 1-0 \cdot 05 \mathrm{~mm}$. mercury.

The importance of eutectics in drying materials from the frozen state has been discussed by Greaves (1954). The materials and concentrations of materials from which freeze-dried plugs can be produced are largely governed by these considerations. A plug which was used extensively in this work was one consisting of peptone and starch. It was found that with the freeze-drying facilities available, peptone alone in concentrations of $5 \%$ or more tended to foam and shrink during drying from the frozen state; only occasionally was a satisfactory drying obtained. The addition of soluble starch, however, in concentration of from 0.25 to $1.5 \%$ consistently resulted in compact plugs. The preparation of such plugs is given below in detail.

Carbohydrates such as glucose are notoriously difficult to dry from the frozen state. However, because glucose has been shown to have remarkable preservative activity for dried bacteria (Fry \& Greaves, 1951), numerous attempts were made to prepare plugs from mixtures of this and other materials. Peptone and glucose, each in about $5 \%$ concentration, were found extremely difficult to freeze-dry, but very occasionally a satisfactory batch was produced. The addition of the polysaccharides dextrin or dextran to mixtures of peptone and glucose, however, consistently resulted in satisfactory drying. Structurally sound plugs were also consistently obtained with dextrin-glucose and dextran-glucose mixtures.

The plugs were all $1.5 \mathrm{ml}$, in volume and dried in the same type of ampoule (PI. 15, fig. 2A). The only solutions which were adjusted for $\mathrm{pH}$ before freezing were those containing ascorbic acid-such mixtures were raised to $\mathrm{pH} 7$.

The full range of plugs which were investigated is shown in Table 2.

Since this work was done a new freeze-drying plant designed by Dr R. I. N. Greaves has been put into operation; by means of it both peptone and peptoneglucose plugs have been dried very efficiently.

Materials used for preparation of plugs

Peptone: Evans bacteriological.

Glucose: B.D.H. Analar, D-glucose. 
Dextrin: Hopkin and Williams (Puriss).

Dextran: 'Intradex' (Glaxo Laboratories Ltd). This is a $6 \%$ salt-free solution of dextran.

Ascorbic acid: Roche Products, Ltd (B.P.).

Starch: B.D.H. Analar, soluble starch.

Ammonium chloride: B.D.H. Ltd.

Thiourea: Hopkin and Williams Ltd.

Serum: Human, inactivated.

\section{Preparation of the peptone-starch plug}

Peptone and starch were added to cold distilled water to give concentrations of 6 and $0.5 \%$ respectively. The mixture was gently agitated over a flame until the starch and peptone dissolved. It was then boiled for a few minutes and volumes of $1.5 \mathrm{ml}$. of the solution were dispensed into sterile ampoules which were then loaded into Kilner jars and stored at $-20^{\circ} \mathrm{C}$. for at least $5 \mathrm{hr}$. The materials were then dehydrated from the frozen state for a period of $18-24 \mathrm{hr}$. The jars were quickly sealed with airtight lids and stored at room temperature until required.

\section{Plugs prepared from other workers' formulae}

(a) Modified 'Mist. desiccans' (Fry \& Greaves, 1951): human serum + 7.5\% glucose (the broth component was omitted-Dr Fry's suggestion).

(b) Modified 'Naylor's dextrin' (Naylor \& Smith, 1946): dextrin 6\%, $\mathrm{NH}_{4} \mathrm{Cl}$ $0.5 \%$, ascorbic acid $0.5 \%$, thiourea $0.5 \%$. (The dextrin content was raised from 2 to $6 \%$.)

(c) Modified 'Naylor's dextran': as above, but dextran instead of dextrin.

\section{Vacuum pump and manifold}

Pl. 15, fig. 1, shows an illustration of the vacuum pump and manifold that were used throughout this work. The vacuum pump ('Speedivac') was that belonging to a small centrifugal freeze-drying unit (Edwards High Vacuum Ltd). The $\mathrm{P}_{2} \mathrm{O}_{5}$ trap was maintained in the well of this unit and was thus rather remote from the manifold which was attached to the side of the well by a short length of pressure tubing. A Pirani gauge was included in the circuit to record pressure changes. The central stem and side arms of the manifold were of stout brass tubing. The pressure tubing sections of the arms of the manifold were equipped with strong spring clips and thus permitted isolation and removal of individual ampoules from the manifold without disturbing the vacuum. Also ampoules could be added to the manifold with only a transient rise of pressure within the system. Rubber nipples (Edwards High Vacuum Ltd) to which the ampoules were attached were fitted into the ends of the pressure tubing.

Another manifold which has also been used for this type of work has been described (Annear, 1956). 


\section{Inoculation of the plugs}

The device shown in Text-fig. 1 was designed for inoculating numbers of plugs in rapid succession with constant (single drop) inocula. The syringe was greased so that the plunger moved smoothly when pressure was applied to it, and the outer wall of the capillary tip was treated with paraffin wax to prevent its being wetted as the drops were expelled. Single drops were discharged by gentle finger pressure on the plunger of the syringe. With a leak-tight rubber connexion between the syringe and pipette, the drops fell cleanly, leaving no fluid beneath the tip. The pipette delivered drops of about $0.02 \mathrm{ml}$. (the exact volume was not important provided that it was constant for each experiment). In one trial, fifteen single drops of a dilute broth suspension of Chromobacterium prodigiosum were discharged on to agar plates and the following data were obtained from the resulting counts: range, 24-36; mean, $31 ; P\left(\chi^{2}\right)=0.97$. Mechanical movement of the plunger by a screw thread was found to be both unnecessary and somewhat inconvenient.

Each plug was inoculated by moving the ampoule up the stem of the pipette until the surface of the plug was about $1 \mathrm{~cm}$. below the orifice of the pipette; the plunger of the syringe was then gently pressed to discharge a drop on the surface of the plug.

\section{Dehydration of the inoculated plugs in vacuo}

Immediate exposure of the inoculated plugs to reduced atmospheric pressure was an important feature of the method, as prolonged contact of the inoculum with the plug resulted in a gradual shrinkage of the soluble material into a mass which became difficult to dehydrate and later difficult to redissolve. Each plug was therefore connected to the manifold as soon as it had been inoculated.

In preliminary experiments it was shown that immediate exposure of inoculated peptone plugs to high vacuum resulted in a mushroom of foam which rose a centimetre or so above the surface of the plug and which often became detached from the main bulk of the plug (Pl. 15, fig. 2B, C). Although the effect of this on the storage survival of organisms was not known, it was felt that a more compact desiccate was desirable. Further experiments showed that if the inoculated plug was subjected

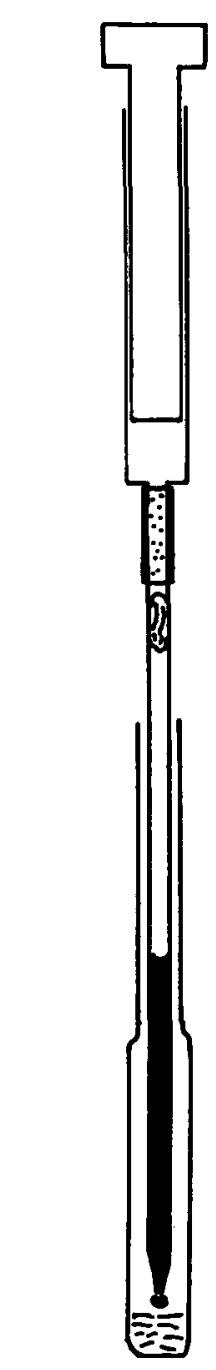

Text-fig. 1. Device for inoculating freezedried plugs. For further explanation, see p. 490. to a preliminary exposure at a higher pressure $(c .10 \mathrm{~mm} . \mathrm{Hg})$, foaming was more gentle and the resultant product more compact; the foam formed a crust only a millimetre or so in depth over the surface of the plug (Pl. 15, fig. 2D). The higher pressure was obtained by arranging a small leak through a glass tap attached to one of the arms of the manifold. A mercury manometer attached to 


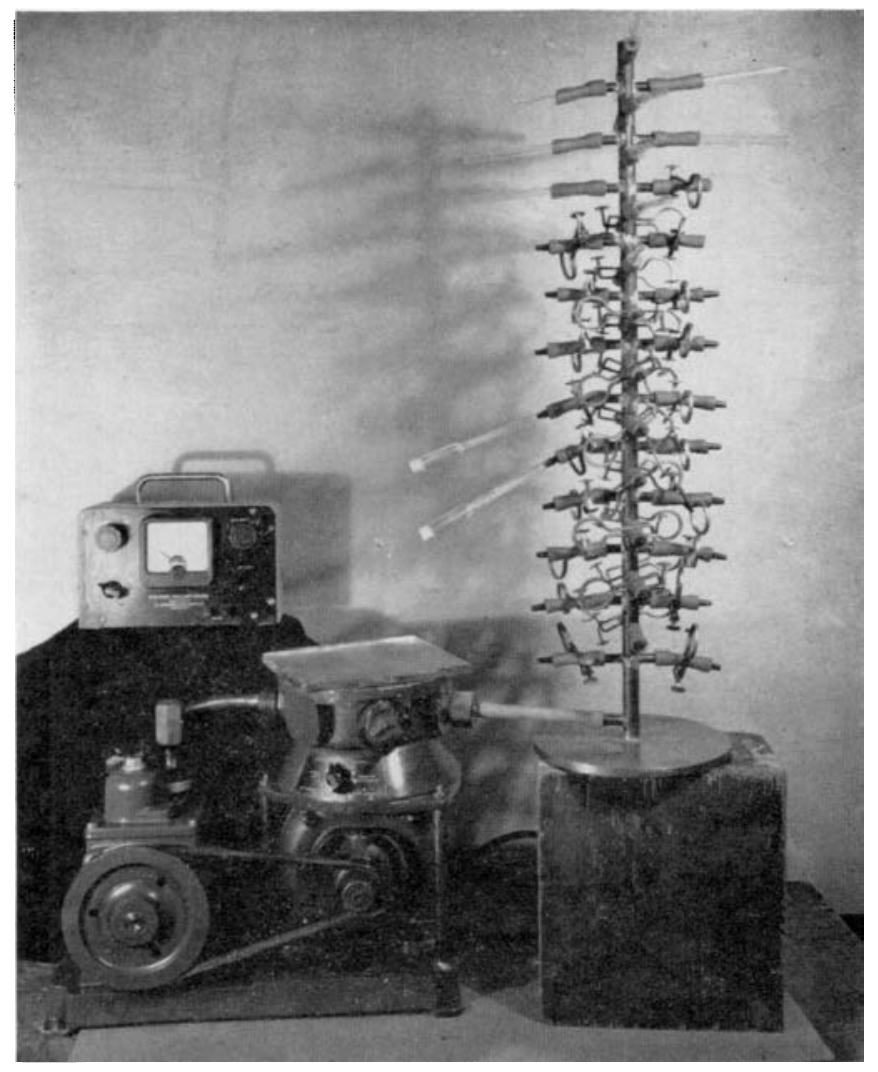

Fig. 1

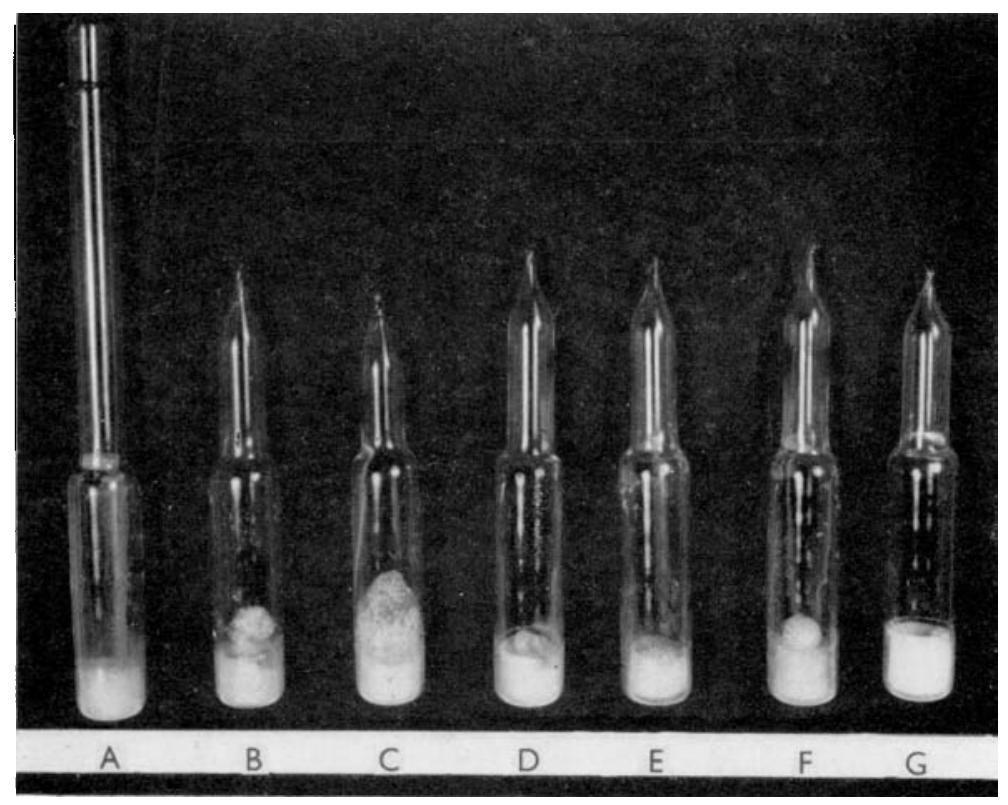

Fig. 2 


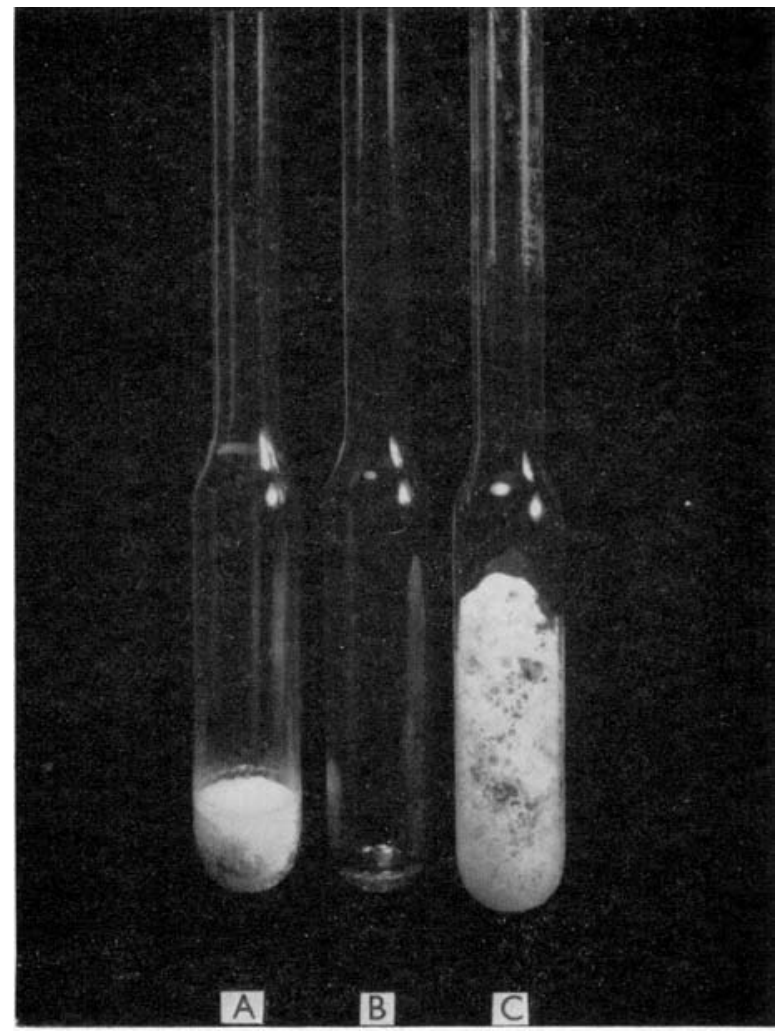


another arm was used to measure the pressure. When a number of plugs were subjected one after another to this treatment, the addition of the second and succeeding ampoules to the manifold resulted in a transient rise of pressure in the system, thus causing partial collapse of the foam in the plugs already there. As the pressure fell, the foam reformed. This process continued until the foam became sufficiently dehydrated to remain compact, regardless of fluctuations of pressure within the manifold. About $5 \mathrm{~min}$. after the last ampoule was connected, the leak was closed to obtain high vacuum; the crusts remained compact and dehydration proceeded rapidly. All the early experiments were done by this two-stage procedure. Although the results from this method seemed satisfactory, it was later felt that the drying procedure would be simpler if the plugs could be exposed to high vacuum immediately after inoculation. It was then shown that the excessive foaming which tended to occur when inoculated plugs were exposed directly to high vacuum could be reduced if the inoculum was allowed to penetrate more deeply into the plug before the ampoule was connected to the manifold. This could be achieved quite simply by tapping the base of the ampoule lightly on the bench after the plug had been inoculated. With this modification, most of the foam was trapped within the plug and the resultant desiccate was reasonably compact. Most of the later experiments were done with this one-stage procedure of dehydration. Pl. 15, fig. $2 \mathrm{E}$ and $\mathrm{F}$ shows the extreme range of foaming which occurred in plugs dehydrated by this simpler procedure.

With materials such as serum or dextran, very little foam is produced with either of the procedures above (Pl. 15, fig. 2G).

The two methods investigated for dehydrating the inoculated plugs are summarized below.

Method 1. A short initial exposure of the plugs to a pressure of $10 \mathrm{~mm}$. followed by prolonged exposure to high vacuum.

Method 2. An immediate and prolonged exposure of the plugs to high vacuum.

At some time during the exposure of desiccates to high vacuum, the ampoules were taken off the manifold, one at a time, constricted, and returned to the manifold. The final step in the procedure was that of sealing the ampoules in vacuo. The ampoules were protected from the light during exposure on the manifold by sleeves of black paper.

\section{Viable counts}

Surface methods of making viable counts were used exclusively. A type of dropping pipette as used by Snyder (1947) and calibrated to deliver drops of $0.02 \mathrm{ml}$. was employed. The pipette was rinsed with boiling water between operations and then rinsed with the suspension to be counted. After the drops had fallen, the plate was held almost vertical and rotated; in this way the drops could be made to spread over an area considerably greater than that which they would cover if the plate were left in a horizontal position as is done in the technique of Miles \& Misra (1938). As a routine no attempt was made to keep the drops separate; the colonies were counted as aggregates of the number of drops delivered on to the plates.

In one trial, twenty-one single drop counts of a dilute broth suspension of Pseudomonas pyocyanea gave the following results: range, 58-88; mean, $72 ; P\left(\chi^{2}\right)=0 \cdot 60$. 
QUANTITATIVE CONSIDERATIONS-AN ILLUSTRATIVE EXPERIMENT

Discussion of the quantitative aspects of this work can best be aided by the description of an experiment which was designed to test the variance of replicate counts on a suspension before drying and the variance of the recoveries of replicate desiccates after drying. When this experiment was done, preliminary information on suitable experimental conditions had been obtained.

\section{METHODS AND MATERIALS}

Organism. Paracolon bacillus (N.C.T.C. 9793).

Culture. Organism grown on nutrient agar for $6 \mathrm{hr}$. at $37^{\circ} \mathrm{C}$.

Suspension. Light suspension made in nutrient broth, then $0.4 \mathrm{ml}$. of this suspension diluted in $100 \mathrm{ml}$. of $10 \%$ glucose.

Plugs. Evans peptone, $6 \%$; starch, $0.5 \%$.

Drying. Method 2 for $1 \mathrm{hr}$.

Recovery medium. Blood-agar.

Counts on undried suspension. For each count, one drop of the glucose suspension was delivered from the inoculating pipette into $5 \mathrm{ml}$. of nutrient broth; one drop volumes of the resultant broth suspensions were delivered on to blood-agar plates ( 3 drops per plate and 2 plates per count).

Counts on desiccates. Each plug was dissolved with $5 \mathrm{ml}$. of broth and counts were made as on the undried suspension.

\section{Proceddue}

(1) Two counts were made on the undried suspension.

(2) Six plugs were inoculated and exposed to high vacuum.

(3) Ten further counts were made on the undried suspension.

(4) After $1 \mathrm{hr}$. drying, the ampoules were taken off the manifold, one at a time, and counts made.

\section{Discussion OF RESUlts}

The counts and a statistical analysis of the counts are presented in Table 1. Also shown are the percentage recoveries after drying for each of the six desiccates. The probability value $P\left(\chi^{2}\right)$ shown in the analysis is extremely satisfactory and reveals the counts as a whole to be a homogeneous group and consistent with Poisson variation. Several important deductions may be made from these results.

(1) The inoculating and dropping pipettes were satisfactory for dispensing small replicate volumes of bacterial suspension.

(2) The analysis failed to reveal any loss of viable organisms after drying.

(3) The organisms in the dissolved desiccates were homogeneously distributed in suspension.

(4) As no significant difference could be shown between the counts made before and those made after the plugs were inoculated, it can be inferred that the number of viable organisms was constant during that period. In other words, each of the plugs received the same inoculum as the $5 \mathrm{ml}$. volumes of broth which were used 
in the counting of the undried suspension. The testing of this feature is a vital consideration in the method. As a routine only one count was made before and one after the inoculation of the plugs. Where significant differences occurred between the two, the suspension was condemned as unstable and the experiment discarded.

Table 1. Analysis of counts of paracolon bacillus in a drying experiment

\begin{tabular}{|c|c|c|c|c|c|c|c|}
\hline \multirow{2}{*}{$\begin{array}{l}\text { Counts } \\
\text { On undried suspension: } \\
\text { Before inoculation of plugs }\end{array}$} & \multicolumn{2}{|c|}{ Means } & $\begin{array}{c}\text { Grand } \\
\text { mean }\end{array}$ & $\chi^{2}$ & D.F. & $P$ & \multirow[t]{2}{*}{$\begin{array}{c}\text { Recovery* } \\
\text { (\%) after } \\
\text { drying }\end{array}$} \\
\hline & $\left.\begin{array}{l}392 \\
415\end{array}\right\}$ & 404 & & & & & \\
\hline After inoculation of plugs & $\begin{array}{l}422 \\
413 \\
450 \\
395 \\
449 \\
426 \\
397 \\
404 \\
401 \\
403\end{array}$ & 416 & 410 & $15 \cdot 7$ & 17 & 0.55 & \\
\hline $\begin{array}{l}\text { On desiccates after drying } \\
\text { for } 1 \mathrm{hr} \text {. }\end{array}$ & $\left.\begin{array}{l}397 \\
409 \\
415 \\
425 \\
398 \\
372\end{array}\right\}$ & 403 & & & & & $\left\{\begin{array}{r}96 \\
98 \\
100 \\
103 \\
96 \\
90\end{array}\right.$ \\
\hline
\end{tabular}

* Calculated from the grand mean of the undried suspension $=414$.

The numbers of colonies per plate (c. 200) were considered to be optimum for this work. To obtain such numbers, previous experience in preparing the suspension was necessary; so also was a knowledge of the expected level of recovery after drying.

It is pertinent to outline here the modifications which would have been introduced in the experiment described, had recoveries of the order of, say, $25 \%$ been expected: $(a)$ the original suspension could have been increased fourfold in concentration and counts made on it from a 1 drop $/ 20 \mathrm{ml}$. dilution or $(b)$ the inocula for the blood plates from the dissolved desiccates could have been increased from 3 drops to 12 drops. In either case, the expected number of colonies on the plates would have been the same as those shown in Table 1.

These hypothetical considerations illustrate the approach that was adopted towards the quantitative procedures in this work. The experiments were designed so that counts could be made directly from the dissolved desiccates. For counts on the undried suspension the volumes of diluent were varied from 5 to $100 \mathrm{ml}$. The numbers of drops per plate were between 1 and 12 . Where the number of organisms in a single drop was expected to be high, sterile diluent was added to the drop on the 
plate so that the organisms were spread over an area from which the colonies were more conveniently and accurately countable.

The lower limit to the recoveries that could be measured by the procedures outlined was of the order of $1 \%$; the investigations here were mainly concerned with recoveries well above this value.

\section{THE RELATIONSHIP BETWEEN GROWTH PHASE OF CULTURE AND RESPONSE TO DRYING}

Numerous systematic studies have shown that bacteria during their logarithmic growth phase (the phase of 'physiological youth') are more sensitive to such treatments as temperature shock and chemical disinfection than organisms in the stationary phase of growth (Dubos, 1949).

No such systematic studies have been made in the field of bacterial preservation, although it is generally held that similar differences in sensitivity are found when bacteria are dried. For example, Fry \& Greaves (1951) showed that a much higher survival was obtained with a $20 \mathrm{hr}$. culture of their paracolon bacillus than with a culture grown for $4 \frac{1}{2} \mathrm{hr}$. The data of Proom \& Hemmons (1949) on this subject are rather difficult to follow, particularly as they refer to $7 \mathrm{hr}$. cultures as being at the beginning of the logarithmic phase and do not mention the temperature at which the organisms were grown.

Attempts have been made here to study the relationship between growth phase and response to drying. Curves of these two variables were obtained for three organisms grown in nutrient broth and for one grown on nutrient agar. Recoveries were measured at the end of the drying period only.

In considering designs for testing these relationships with broth cultures it was evident that two approaches were possible. (1) Samples from the culture could be dried without preliminary dilution; such a procedure would involve a wide variation in the numbers of organisms that were dried at the various stages of growth. (2) Samples of the culture could be diluted so that approximately the same numbers could be dried at the various stages of growth. The latter alternative was chosen for the experiments described here.

It is not easy to measure growth rates of organisms on agar surfaces and thus relate to them recoveries after drying, but at least these recoveries can be related to the age of such cultures. This was done for one organism.

Before the experiments described in this section were done a considerable number of preliminary trials were made to provide information for suitable experimental conditions.

\section{METHODS}

Experiments with nutrient broth cultures

The organisms studied were Staphylococcus aureus, Vibrio cholerae (624) and the paracolon bacillus (N.C.T.C. 9793). The inocula for the experiments were obtained from $18 \mathrm{hr}$. broth cultures grown at $37^{\circ} \mathrm{C}$. (i.e. cultures in the stationary phase).

The experiments were done in flasks containing $200 \mathrm{ml}$. of broth through which air was bubbled. The vibrio and the paracolon bacillus were grown at $31^{\circ} \mathrm{C}$. and 
the staphylococcus at $33^{\circ} \mathrm{C}$. At various intervals samples were withdrawn and diluted in $10 \%$ glucose (suspending medium). Plugs (peptone, 6\%; starch, $0.5 \%$ ) were inoculated with one drop volumes of these suspensions and exposed directly to high vacuum (drying method 2). At the same time the suspensions were counted to provide figures for the construction of the growth curves and for the calculation of recoveries after drying.

Counts were made from the desiccates after a drying period of $24 \mathrm{hr}$. All counts were made on blood-agar.

\section{Experiment with nutrient agar culture}

The test organism was Vibrio cholerae (624). A nutrient agar plate was flooded with a dilute suspension of the vibrio which was obtained from a broth culture in its stationary phase and the plate was incubated at $37^{\circ} \mathrm{C}$. As soon as a visible film of growth appeared, and at intervals thereafter, growth was scraped from the surface and emulsified in broth. Dilutions $(1 / 250)$ of the broth suspensions were made in $10 \%$ glucose, and one drop volumes of these secondary suspensions were dried under the same conditions as those in the nutrient broth experiments, except that the inoculated plugs were dried for $1 \mathrm{hr}$. only. All counts were made on blood agar.

\section{Results}

\section{Experiment with nutrient broth cultures}

The growth curves and recoveries after drying, both plotted logarithmically, are shown in Text-fig. 2; there were wide differences in the growth-phase recovery patterns for the three organisms. In the case of the staphylococcus no marked difference occurred at any stage during the experiment between the counts before and after drying. The vibrio showed an approximate thirty-fold ratio between the highest recovery $(38 \%)$ and the lowest $(1.4 \%)$. With the paracolon bacillus, the ratio was about fivefold (' 100 ' and $23 \%$ ). The lowest recoveries of both the vibrio and the paracolon bacillus after drying were obtained during the logarithmic phase of growth.

An interesting difference between the vibrio and the paracolon bacillus was shown during the lag phase of growth. In the case of the vibrio, a steep decline in recoveries after drying progressed with this phase, whereas no marked differences were apparent in this phase with the paracolon bacillus; it was not until the organisms had begun to multiply that a decline in recoveries after drying occurred.

\section{Experiment with nutrient agar culture}

The results from the experiment are shown in Text-fig. 3. The recoveries from the first two samplings were markedly lower than those from the succeeding ones and the change from the lower to the higher recoveries was extremely rapid. The similarity between these results and those obtained with the nutrient broth culture was quite marked. 


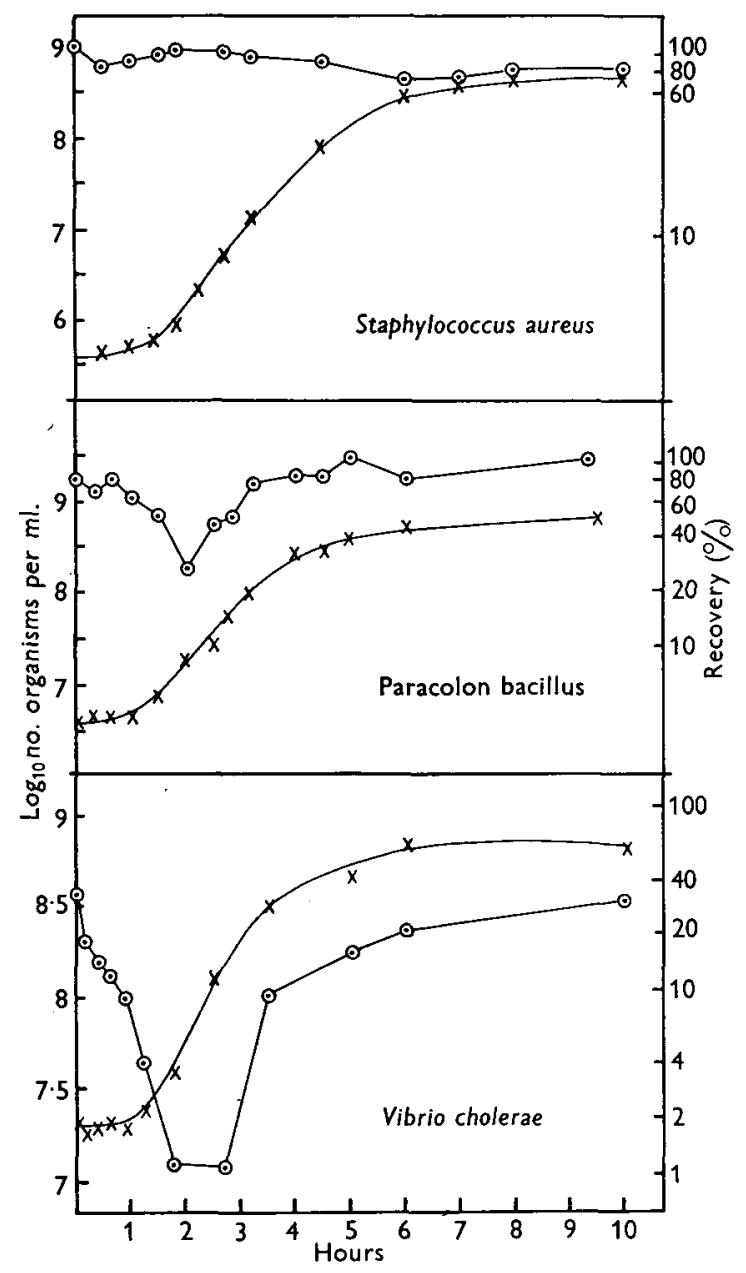

Text-fig. 2. Relationship for three organisms between growth and recoveries after drying. Experiments with nutrient broth cultures. $x-x, \log _{10}$ number of organisms/ml.; $\odot-\odot$, recoveries after drying.

\section{Discussion}

It is apparent that, with the drying technique under investigation, all bacteria do not conform to a general pattern with respect to the relationship between their phase of growth and their recoveries after drying. It would be of great interest to determine such patterns for a number of other organisms, as such information might well indicate the reasons for these differences.

In the experiments shown in Text-fig. 2, observations were made for about the first $10 \mathrm{hr}$. of growth; other experiments not recorded here showed that no significant changes in the curves of growth or recoveries after drying occurred for at least a further $12 \mathrm{hr}$. No observations were made beyond this period.

The available evidence suggests that from a practical point of view the stationary phase is the most reliable period for drying bacteria. 
The curves obtained for Vibrio cholerae and the paracolon bacillus are very similar to one published by Hegarty \& Weeks (1940), who studied the relationship between the growth phase of Escherichia coli and the sensitivity of the organism to cold shock.

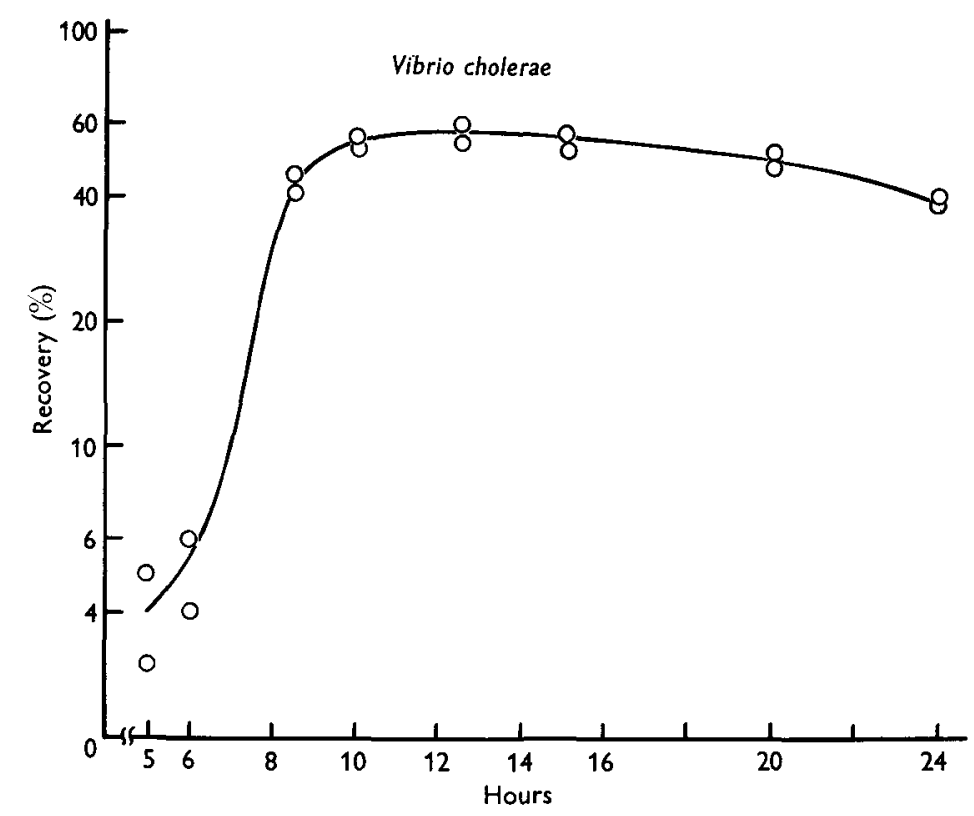

Text-fig. 3. Relationship for Vibrio cholerae (624) between period of growth on nutrient agar and recoveries after drying.

\section{GENERAL STUDIES ON THE PRESERVATION OF NEISSERIAE AND VIBRIOS}

The factors which have been studied most extensively here in relation to the drying of neisseriae and vibrios are the compositions of the plugs and the suspending media. In any one experiment it was a simple matter to inoculate a variety of plugs with the one suspension of organisms and so obtain strictly comparable results. It should be remembered that the final medium in which the organisms were preserved was derived both from the plug and from the inoculum.

In addition to the above factors, there is some information on the storage temperature and the period of drying as they affect the survival of the organisms.

\section{METHODS}

Growth of parent cultures. The vibrios were grown on nutrient agar at $30^{\circ} \mathrm{C}$. for $20 \mathrm{hr}$. and the neisseriae on chocolate agar at $37^{\circ} \mathrm{C}$. for $24 \mathrm{hr}$.

Suspensions. A primary suspension of the organisms was made in nutrient broth by rubbing a charged loop on the wall of a tube of broth and gradually washing the emulsified growth into the bulk of the broth. The broth suspension was then shaken vigorously by hand and the final suspension made by diluting the broth suspensions $1 / 200-1 / 400$ in the test suspending medium. The numbers 
Table 2. Recoveries after drying of various species of Neisseria and Vibrio

\begin{tabular}{|c|c|c|c|c|c|c|c|c|c|c|c|c|}
\hline Organism & 齐 & $\begin{array}{c}0 \\
9 \\
0\end{array}$ & $\stackrel{\vec{a}}{\vec{a}}$ & $\stackrel{B}{A}$ & $\dot{B}$ & $\stackrel{0}{0}$ & 0 & $1-4$ & ecove & $1 \%$ & 12 & $10-29$ \\
\hline N. gonorrhoeae & $1 a$ & $c$ & D 72 & 1 & $\mathbf{3}$ & Room & 66 & 53 & - & - & 57 & - \\
\hline (N.C.T.C. 8375) & $1 b$ & $c$ & F 73 & 1 & 3 & Room & 61 & 41 & - & - & 41 & - \\
\hline & $1 c$ & $c$ & G 74 & 1 & 3 & Room & 59 & 33 & - & - & 27 & - \\
\hline & 2 & $g$ & F 68 & 1 & 2 & Room & 51 & - & - & 一 & 52 & 25 \\
\hline & 3 & $c$ & D 85 & 1 & 6 & Room & 63 & - & - & - & - & 45 \\
\hline N. gonorrhoeae & $4 a$ & $f$ & E 190 & 2 & 18 & Room & 一 & - & 36 & 38 & 20 & \\
\hline$(32619)$ & $4 b$ & $f$ & E 190 & 2 & 18 & $4^{\circ} \mathrm{C}$ & - & - & 51 & 55 & 40 & $40^{*}$ \\
\hline & $4 c$ & $f$ & E 190 & 2 & 18 & $37^{\circ} \mathrm{C}$. & 一 & - & 0.7 & $0 \cdot 3$ & - & 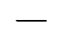 \\
\hline N. gonorrhoeae & $5 a$ & $c$ & A 89 & 1 & 6 & Room & 62 & - & - & 41 & - & - \\
\hline (P.D.) (2695) & $5 b$ & $c$ & D 91 & 1 & 6 & Room & 69 & 一 & - & 45 & 一 & - \\
\hline & $6 a$ & $c$ & D 115 & 2 & 3 & Room & 70 & 36 & - & - & 30 & 31 \\
\hline & $6 b$ & $c$ & U 118 & 2 & 3 & Room & 55 & 43 & - & - & 45 & 28 \\
\hline & $7 a$ & $c$ & E 130 & 2 & 3 & Room & 41 & - & 37 & - & 44 & 33 \\
\hline & $7 b$ & $c$ & H 133 & 2 & 3 & Room & 69 & - & 56 & - & 50 & 50 \\
\hline & $8 a$ & $c$ & E 130 & $\mathbf{l}$ & 3 & Room & 56 & - & - & - & 63 & 29 \\
\hline & $8 b$ & $c$ & H 133 & 1 & 3 & Room & 59 & - & 一 & 一 & 64 & 27 \\
\hline N. meningitidis & $9 a$ & $c$ & D 72 & 1 & 1 & Room & 75 & 68 & - & - & 55 & 一 \\
\hline (N.C.T.C. 8365) & $9 b$ & $c$ & F 73 & 1 & 1 & Room & 70 & 60 & - & - & 44 & - \\
\hline & $9 c$ & $c$ & G 74 & 1 & 1 & Room & - & 57 & - & - & 30 & - \\
\hline V. cholerae (624) & $10 a$ & $b$ & U 108 & 2 & 7 & Room & 29 & 一 & - & 18 & 13 & 一 \\
\hline (Ogawa strain) & $10 b$ & $b$ & D 107 & 2 & 7 & Room & 16 & 一 & - & 2 & $\mathbf{3}$ & - \\
\hline (R.M.F.) & $10 c$ & $b$ & D 115 & 2 & 7 & Room & 24 & - & - & 3 & 2 & - \\
\hline & $11 a$ & $d$ & R 148 & 2 & 24 & Room & 16 & 9 & 一 & - & - & - \\
\hline & $11 b$ & $d$ & E 154 & 2 & 24 & Room & 62 & 38 & - & 22 & - & 18 \\
\hline & $11 c$ & $d$ & S 162 & 2 & 24 & Room & 53 & 16 & - & - & - & 9 \\
\hline & $11 d$ & $d$ & L 163 & 2 & 24 & Room & 47 & 14 & - & 18 & - & 6 \\
\hline & $11 e$ & $d$ & P 165 & 2 & 24 & Room & 42 & 40 & - & 49 & 一 & 42 \\
\hline & $11 f$ & $d$ & V 167 & 2 & 24 & Room & 58 & 40 & - & 38 & - & 36 \\
\hline & $12 a$ & $d$ & L 163 & 2 & 7 & Room & 48 & - & - & 7 & - & 5 \\
\hline & $12 b$ & $d$ & P 165 & 2 & 7 & Room & 54 & 41 & - & 47 & - & 46 \\
\hline & $12 c$ & $d$ & V 167 & 2 & 7 & Room & 50 & 28 & - & 37 & - & 24 \\
\hline & $12 d$ & $d$ & E 169 & 2 & 7 & Room & $\mathbf{5 5}$ & 20 & - & 22 & - & 26 \\
\hline & $12 e$ & $d$ & E 169 & 2 & 7 & $4^{\circ} \mathrm{C}$ & - & 47 & - & 54 & - & 43 \\
\hline & $13 a$ & $d$ & P 165 & 2 & 24 & Room & 58 & 39 & - & 41 & - & 33 \\
\hline & $13 b$ & $d$ & E 169 & 2 & 24 & Room & 53 & 26 & - & 30 & - & 20 \\
\hline & $13 c$ & $d$ & O 174 & 2 & 24 & Room & 68 & 48 & - & - & - & - \\
\hline & $13 d$ & $d$ & O 174 & 2 & 24 & $4^{\circ} \mathrm{C}$ & - & 63 & - & - & - & 58 \\
\hline & $14 a$ & $d$ & E 169 & 2 & 20 & Room & 55 & 23 & - & 20 & - & - \\
\hline & $14 b$ & $d$ & A 181 & 2 & 20 & Room & 42 & 25 & 一 & 26 & - & - \\
\hline & $14 c$ & $d$ & O 182 & 2 & 20 & Room & 56 & 一 & - & 50 & - & - \\
\hline & $15 a$ & $d$ & E 169 & 2 & 24 & Room & 41 & 20 & - & 24 & - & 15 \\
\hline & $15 b$ & $d$ & M 183 & 2 & 24 & Room & 37 & - & 一 & 6 & - & - \\
\hline & $15 c$ & $d$ & N 184 & 2 & 24 & Room & 40 & 7 & - & 1 & - & \\
\hline
\end{tabular}




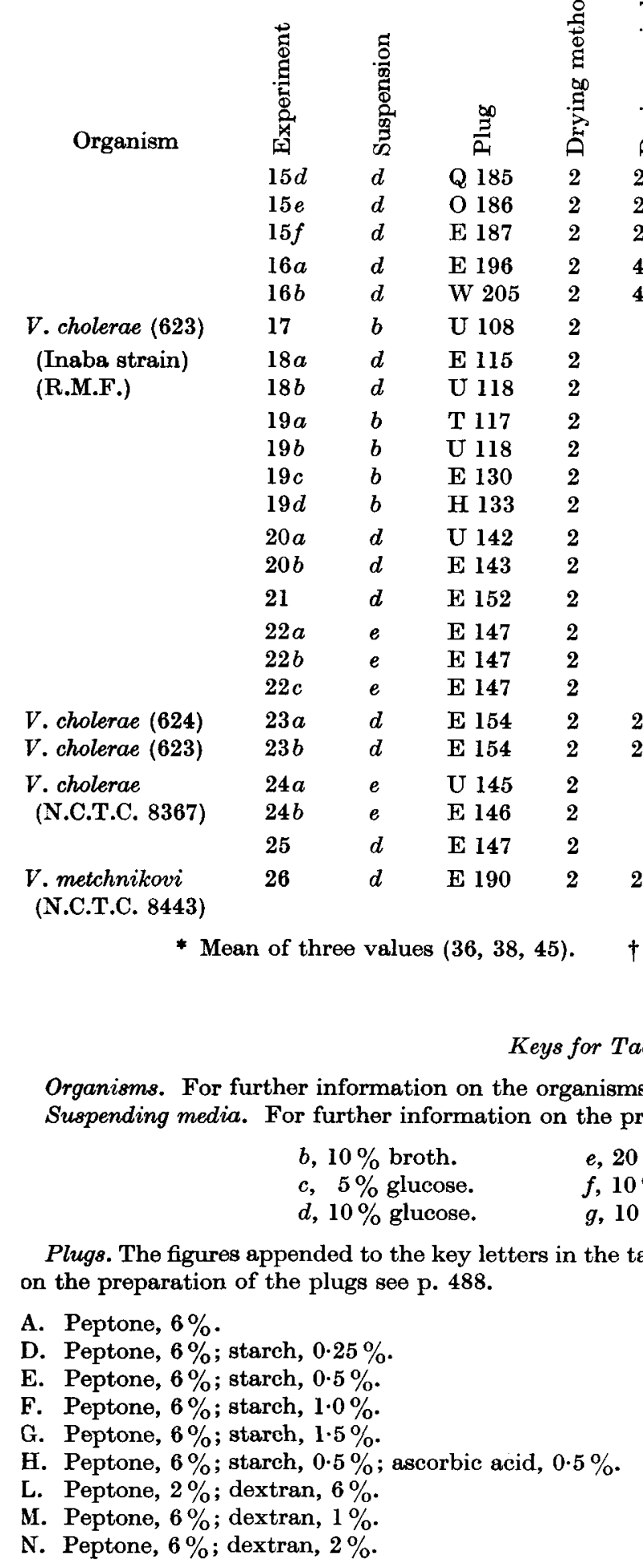

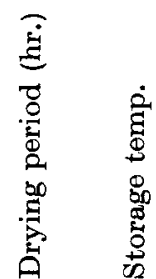

Room $44 \quad 47-55-36$

Room $59 \quad-\quad-35 \quad-$

Room $51 \quad 15-14-$

$\begin{array}{lllllll}\text { Room } & 47 & 17 & 18 & - & -\end{array}$

$\begin{array}{llllll}\text { Room } & 49 & 27 & 29 & - & -\end{array}$

Room $52 \quad 29 \quad-\quad 13 \quad-\quad-$

Room $22 \quad 26 \quad-\quad 16 \quad-$

Room $44 \quad 42$

Room 17

Room 30

Room 21

Room 18

Room 36

Room 35

Room 20

$4^{\circ} \mathrm{C}$.

Room 12

$37^{\circ} \mathrm{C}$. -

Room 52

Room 33

Room 2

Room 21

Room 21

Room 25
Storage (months)

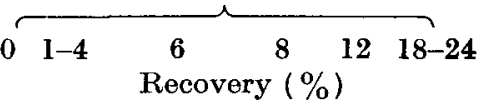

\begin{tabular}{l} 
- \\
\hline \\
\hline \\
\hline \\
\hline \\
1 \\
8 \\
1 \\
1 \\
9 \\
1 \\
1
\end{tabular}

Mean of three values (13, 15, 17).

Method of drying. For description of the two methods (1 and 2) see p. 490. 
of organisms in the one-drop inocula were of the order of $10^{4}-10^{5}$. A list of the suspending fluids investigated is shown in Table 2.

Plugs. A list of the freeze-dried plugs investigated is shown in Table 2.

Method and period of drying. Both methods ( 1 and 2) of dehydrating the inoculated plugs were used. Ampoules were sealed after various periods of exposure on the manifold. In some experiments counts were made on desiccates taken directly from the manifold.

Storage of desiccates. All desiccates were stored in vacuo in the dark and either at $4^{\circ} \mathrm{C}, 37^{\circ} \mathrm{C}$. or room temperature (range $c .15-27^{\circ} \mathrm{C}$.).

Recovery media. Counts of the vibrios were made on blood-agar plates which were incubated at $30^{\circ} \mathrm{C}$. Counts of the neisseriae were made on chocolate agar plates which were incubated at $37^{\circ} \mathrm{C}$. in jars the atmospheres of which were saturated with water vapour and charged with $\mathrm{CO}_{2}$ from candles.

\section{Experimental proceddre}

The procedure which was followed for the experiments described here is outlined below:

1. Pump set running and manifold connected.

2. Bacterial suspension prepared.

3. First count made on bacterial suspension.

4. Plugs inoculated and exposed to vacuum dehydration.

5. Second count made on bacterial suspension.

6. Ampoules taken from manifold one at a time, constricted and returned to high vacuum.

7. Ampoules sealed off in vacuo.

8. Counts made on desiccates. These were made either on desiccates taken directly from manifold or on desiccates sealed and stored in vacuo. In the former case it was not necessary to constrict the ampoules.

\section{Results}

The results of these investigations are shown in Tables 2, 3 and 4 and in Textfig. 4. Most of the data are in the twenty-six experiments shown in Table 2; in the text, reference is made to these experiments without mentioning the table by number.

Table 3. Recoveries of Vibro cholerae (624) after drying in four different batches of peptone-starch plugs for 1 and for $24 \mathrm{hr}$.

Suspending medium: $d(10 \%$ glucose $)$.

Plugs: E (Evans peptone, $6 \%$; starch, $0.5 \%$ ).

Drying: Method 2.

Batch no.
of plugs
190
233
234
209

Dried, $1 \mathrm{hr}$.

$\begin{array}{lc}\text { Recoveries (\%) } & \text { Mean } \\ 57,64,65,58 & 61 \\ 62,52,61,54 & 57 \\ \text { Not done } & - \\ \text { Not done } & -\end{array}$

$\begin{array}{cc}\overbrace{\text { Recoveries }(\%)}^{\text {Dried, } 24 \mathrm{hr} .} & \\ 54,53,53,56 & \text { Mean } \\ 55,53,54,48 & 54 \\ 46,53,55,52 & 53 \\ 45,44,44 & 51 \\ & 44\end{array}$


Table 4. Observed and expected frequencies of probability $(P)$ values from $\chi^{2}$ analyses of ninety-four pairs of counts of Vibrio cholerae (624) on blood-agar

The pairs of counts were composed of two members: $(a)$ those counts made before the plugs were inoculated; $(b)$ those counts made after the plugs were inoculated.

\begin{tabular}{cr} 
Suspending medium: $10 \%$ glucose & 79 pairs \\
$10 \%$ broth & 8 pairs \\
$10 \%$ glucose $+10 \%$ broth & 7 pairs \\
\cline { 2 - 2 } & 94 pairs
\end{tabular}

$\begin{array}{cccc}\text { Range of } P & \text { Observed } & \text { Expected } & \frac{(\text { Obs. }- \text { Exp. })^{2}}{\text { Exp. }} \\ <0 \cdot 01 & 2 & 0.94 & 1 \cdot 19 \\ 0 \cdot 01-0 \cdot 1 & 12 & 8 \cdot 46 & 1 \cdot 48 \\ 0 \cdot 1-0.5 & 38 & 37.60 & 0 \cdot 00 \\ 0.5-0.9 & 34 & 37.60 & 0.31 \\ 0.9-0.99 & 6 & 8.46 & 0 \cdot 71 \\ >0.99 & 2 & 0.94 & 1 \cdot 19 \\ \text { Totals } & 94 & 94.00 & 4.88\end{array}$

$\chi^{2}=4 \cdot 88$. D.F. $=5 . \quad P($ goodness of $\mathrm{fit})=0 \cdot 42$.

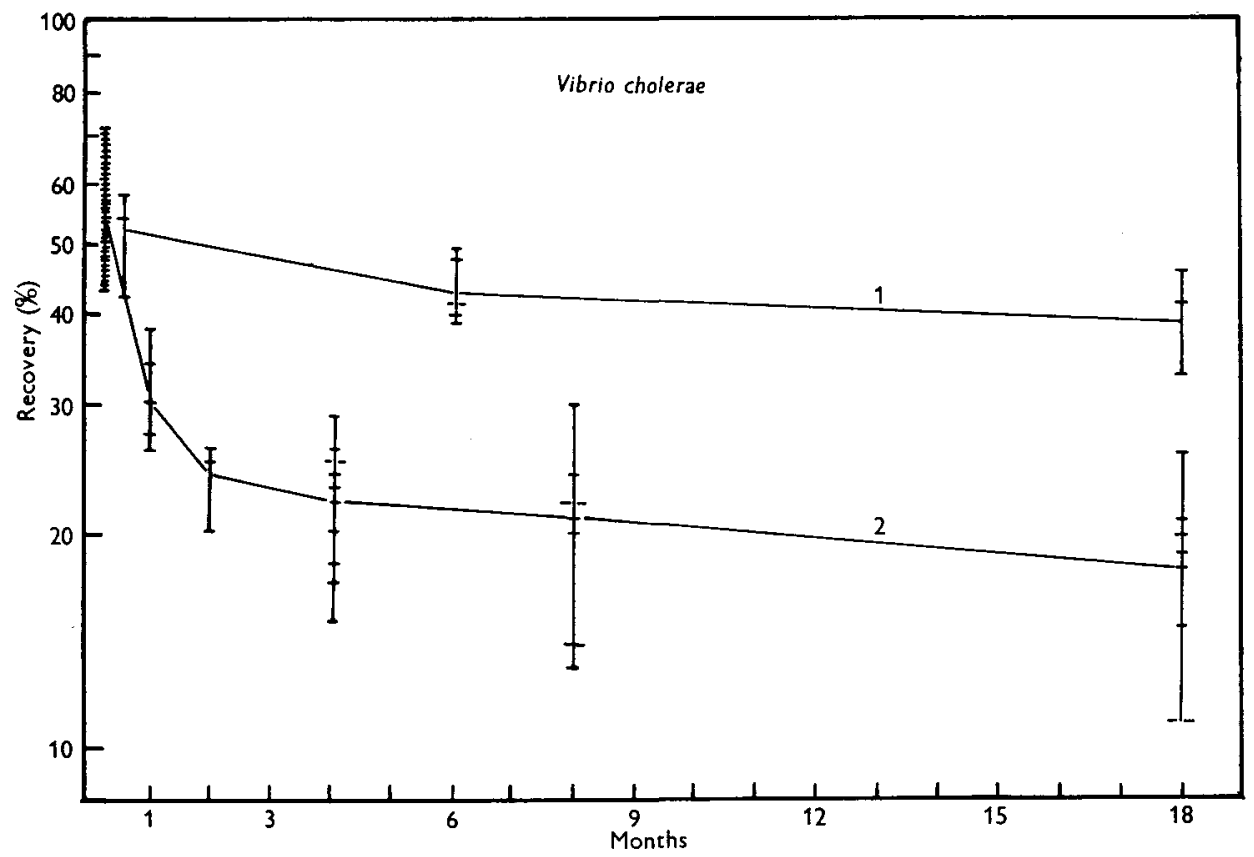

Text-fig. 4. Recoveries in numerous experiments of Vibrio cholerae (624) immediately after drying and after storage. There are fifty-nine points for the first set of values in curve 2. Many of the experiments are to be found in Table 2. Suspending medium was $10 \%$ glucose for all experiments. Plugs for curve 1 were: peptone, $2 \%$; dextran, $6 \%$; glucose, $5 \%$. Plugs for curve 2 were: peptone, $6 \%$; starch, $0.5 \%$. 


\section{Analysis of Results}

\section{Variations among results}

(a) Replicate counts on undried suspensions. Each suspension which was dried was counted in duplicate (see p. 492). A satisfactory agreement between the two counts was obtained for each of the experiments shown in Table 2. For Vibrio cholerae (624) it was possible to collect and analyse ninety-four pairs of these counts; the results (Table 4) show a very good agreement with the expected (Poisson) variance.

(b) Replicate desiccates—counted immediately after drying. These variations among the results were satisfactorily small. The several examples shown in Tables 1 and 3 have no more than a reasonable scatter.

(c) Replicate desiccates-counted after storage. Only two sets of stored desiccates were tested. The results, shown at the base of Table 2 , show satisfactory agreement within each set.

(d) Batches of peptone plugs. Four batches of peptone-starch plugs made from the same stock reagents were tested in parallel; the results are shown in Table 3. Except for the one slightly divergent set of recoveries, for which no explanation can be offered, there was good agreement between the batches.

Different brands or different batches of the same brand of peptone were not tested.

(e) Similar experiments. Text-fig. 4 shows some results from two groups, each of similar experiments, with $V$. cholerae (624). The scatter of the few values shown in curve 1, where the recoveries are high, is quite small. In curve 2, where the recoveries were lower, the scatter has increased with the storage period; at the last point of observation (18 months) the highest recovery is a little more than twice the lowest. This diversity is far more than is desirable and was probably the result of many factors including those of the foam composition and structure of the desiccates (see section on methods of drying, p. 502).

$(f)$ Different organisms dried under similar conditions. Only on one occasion (Exp. 23), was more than one organism dried in the same experiment. In this instance, one suspension was counted, and inoculated plugs from it were connected to the manifold. Then the procedure was repeated for the second organism. Although there was a considerable difference between the first two recoveries, the remainder were remarkably similar. The available evidence permits little in the way of comparisons between organisms, but does suggest that among the vibrios, $V$. metchnikovi was the most sensitive to the procedures followed.

(g) Miscellaneous. In a few experiments (e.g. Exp. 20) significantly lower recoveries were obtained from earlier stored desiccates than from later ones. The reason for these anomalies is unknown, but variations in the recovery media or in some aspect of the counting technique seem the most likely explanation.

\section{Method of drying}

No direct comparisons of the two methods of drying have been made, but both methods gave satisfactory results with the neisseriae. Only Method 2 was used for drying the vibrios. 
The survival of the organisms would certainly be influenced by the composition and structure of the foam in the inoculated and dried plugs, and these features are undoubtedly modified by the degree of mixing which occurs between the inoculum and the solid peptone before the ampoule is exposed to low pressure. It would seem impossible to control these factors rigidly, and variations in recoveries after drying may be partly due to them.

\section{Period of drying}

It was shown by Fry \& Greaves (1951) and by Record \& Taylor (1953) that where glucose is present in the desiccates it is possible to ensure high survival of bacteria with enormous latitude in the period of drying. Such are the conclusions that have been drawn here, where, as may be seen from Table 2, recoveries from comparable desiccates dried for widely different periods were reasonably similar. Also Table 3 shows two sets of recoveries of $V$. cholerae (624) after drying for 1 and for $24 \mathrm{hr}$; the difference between the two sets is very slight. Unfortunately, there are no data from stored desiccates which were subjected to varying periods of pumping in the one experiment.

\section{Peptone and starch}

In the two experiments (Exps. 5 and 14) where plugs of peptone alone were used, glucose was present in the inocula so assessment of peptone alone as a preservative has not been possible.

In Exps. 1 and 9, peptone plugs with three starch contents were tested; in both experiments markedly different recoveries were obtained after storage, particularly between desiccates with the lowest and highest starch contents.

In Exp. 5 ( $N$. gonorrhoeae), recoveries were similar from two batches of peptone plugs, one with no added starch and the other with a starch content of $0 \cdot 25 \%$. Similar results may be seen for $V$. cholerae in Exp. 14.

Where peptone and glucose were present, it appeared that starch, at concentrations of $0.5 \%$ or less, had no effect, one way or the other. This concentration of starch $(0.5 \%)$ was sufficient to stabilize peptone during freeze-drying.

\section{Glucose}

Where broth suspensions of $V$. cholerae were dried in peptone plugs (Exps. $10 b$ and $10 \mathrm{c}$ ), very low recoveries were obtained. In similar experiments where the organisms were suspended in $10 \%$ glucose, much higher recoveries resulted (Textfig. 4 and numerous experiments in Table 2), but the highest recoveries were from those experiments where glucose was present in both inoculum and plug (Exps. $13 c, 13 d$ and 14c). There can be no doubt that glucose is an extremely effective preservative for the vibrios, and the findings of Fry \& Greaves (1951) with respect to this substance have been amply confirmed.

The effect of glucose on the drying of the neisseriae cannot be determined from the experiments reported here because the sugar was present in all desiccates. However, these organisms seem far less dependent upon it than do the vibrios for survival during storage. 


\section{Dextran and dextrin}

Where $V$. cholerae was suspended in $10 \%$ glucose and dropped on plugs of mixtures of dextran, peptone and glucose (Exps. 11e, 12b,13a and 15d) high recoveries were obtained (see also Text-fig. 4). These recoveries, however, were very similar to those from desiccates in which the dextran component was absent (Exps. 13c, 13d, 14c and 15e). In the absence of peptone (Exps. $11 a$ and $11 c$ ) the recoveries, particularly after storage, were relatively low. Also in the absence of 'adequate' concentrations of glucose (Exps. $15 a, 15 b$ and $15 c$ ), dextran appeared to depress the preservative effect of peptone.

It seems highly likely that for $V$. cholerae, dextran (i.e. 'Intradex') had little preservative activity. It was, however, a very effective stabilizing agent during the freeze-drying of mixtures of peptone and glucose. Moreover, it had no adverse effect when 'adequate' concentrations of glucose and peptone were present.

Very low recoveries were obtained when a broth suspension of $V$. cholerae was dried in dextrin-glucose plugs (Exp. 19a)

\section{Ascorbic acid}

In Exp. 19 a suspension of $V$. cholerae in dilute broth was dried in peptonestarch plugs with and without $0.5 \%$ ascorbic acid, with almost identical results. In Exps. 7 and 8 with $N$. gonorrhoeae suspended in $5 \%$ glucose, the results were somewhat equivocal. It is obvious, however, that in the experiments here, ascorbic acid had nothing approaching the effect obtained by Stamp (1947). The activity of this substance may, however, be markedly modified by the other ingredients-Stamp's experiments were carried out in gelatine.

\section{Modified 'Naylor's media'}

With $V$. cholerae higher recoveries were obtained in modified 'Naylor's dextrin' plugs where the organisms were suspended in $10 \%$ glucose than where suspended in $10 \%$ broth (Exps. 17, 18, 19 and 20). Effective preservation of N. gonorrhoeae was obtained when a suspension of organisms in $5 \%$ glucose was dried in modified 'Naylor's dextrin' (Exp. 6).

Where suspensions of $V$. cholerae in $10 \%$ glucose were dried in plugs of modified 'Naylor's dextran' (Exps. $11 f$ and $12 c$ ) very reasonable recoveries were obtained.

In general, the evidence suggests that glucose improves the preservative activity of the reagents in the media modified from the formula of Naylor \& Smith. Indeed, it would be of great interest to determine whether or not the ascorbic acid and thiourea could be replaced by glucose. There appears to be little if any difference between dextrin and dextran as the basic constituent of the modified NaylorSmith media.

It is of interest that high recoveries of $V$. cholerae and $N$. gonorrhoeae were obtained after drying in materials free from protein or protein residues. 


\section{Modified 'Mist. desiccans'}

Very satisfactory results were obtained in the one trial (Exp. 16) of this medium, but recoveries as high or higher were obtained in plugs which could be more easily prepared, so no further work was done with it.

\section{Storage temperature}

In Exps. 4, 12, 13 and 22, where the effect of storage temperature was tested on the desiccates, it may be seen that consistent differences were obtained between the recoveries. Storage at $37^{\circ} \mathrm{C}$. proved highly destructive. The results strongly suggest that storage at 'room' temperature in hot climates would be most undesirable, and that 'low' temperature storage in temperate as well as hot climates is advisable.

\section{GENERAL DISCUSSION}

Freeze-drying techniques for preserving bacteria can be controlled with respect to such variables as temperature and rate of water removal (Hutton, Hilmoe \& Roberts, 1951; Greiff \& Pinkerton, 1954). By contrast, the technique investigated here was characterized by no such precision, nor does it seem likely that it can be. Nevertheless, the results indicate that a reasonable standardization was achieved.

The degree of cooling which occurs during the dehydration of the inoculated plugs is unknown, but it seems most unlikely that the highly concentrated solutions of peptone would freeze, even transiently, under reduced pressure. The technique may best be described as drying from the viscous state, dehydration beginning at a stage where the ratio between water and solids is relatively low.

The results presented here give grounds for believing that the method is at least as efficient as and in most cases more efficient than previous methods. The rapidity with which water is removed from the bacterial suspension and the efficacy of the preserving materials seem the most likely explanations for the higher recoveries obtained. The viable count, however, is the basis upon which drying methods are assessed and compared, and unless workers have used strictly comparable methods for carrying out these counts the differences in efficiency between their methods may be more apparent than real. It may be more than coincidence, for example, that the gonococcus, which has hitherto given such low recoveries, is also extremely fastidious in its growth requirements.

A variety of materials and mixtures have been investigated here for preservative activity. Good results were obtained with modifications of 'Mist. desiccans' (Fry \& Greaves, 1951) and the formula advocated by Naylor \& Smith (1946), but the substances which have been tested most extensively, with which the best results were obtained, and which are well worth further study, are peptone and glucose.

Peptone has been used with conspicuous success by Collier (1955) for the preservation of smallpox vaccine. He found it superior to serum and bovine plasma albumen and suggested that preservative activity might be related to molecular size. A study of peptone as a preservative for bacteria was made by Heller (1941), who found it more effective than aluminium hydroxide, starch, gum-tragacanth 
and gastric mucin. He was able to relate the preservative activity of these substances to their gold numbers which indicate hydrophilic activity or the ability of the colloid to protect another from the precipitating action of an electrolyte. This attractive hypothesis needs further investigation.

No further light has been shed on the mechanism of the protective effect of glucose, but it appears that where peptone is present, organisms may vary considerably in their glucose requirement for effective preservation. Although the presence of glucose in the drying medium has been shown to be of great importance in the preservation of the vibrios, particularly for stable storage, there has been no indication that the concentration necessary for high immediate recoveries after drying is critical.

A feature of the drying procedure which requires investigation is a study of the losses, if any, which occur during the brief interval between the inoculation of the plugs and their exposure to high vacuum. This is rather a difficult problem, as it was found that the inoculated but undried plugs were very difficult to redissolve (whereas dried, inoculated plugs were very readily dissolved).

One limitation of the present method of drying is that it can cope only with small volumes of microbial suspensions. Although the limit to the number of organisms that can be contained in a 'drop' volume is well above that necessary for the maintenance of stock cultures, it may be below that suitable for the preservation of living vaccines.

In all procedures for drying organisms and recovering them from the dried state there are treatments other than dehydration, per se, to which the organisms are subjected (e.g. freezing and rehydration). Unless the effects of these treatments are known, it is unwise and possibly untrue to use the phrase 'sensitive to drying', as is so often done, with respect to organisms which yield low recovery values in a preservation procedure.

The results of this investigation encourage the belief that for the preservation of micro-organisms, further consideration should be given to drying methods in which the removal of water is carried out rapidly and from unfrozen preparations. A similar viewpoint was expressed by Frobisher, Parsons, Pai \& Hakim (1947).

\section{SUMMARY}

1. A new method for the preservation of bacteria by drying is described.

2. Marked differences between organisms were found with respect to the relationship between growth phase and recoveries after drying.

3. Recoveries of several strains of neisseriae and vibrios, both immediately after drying and after storage, were considerably higher than any previously recorded for similar species of these genera.

\section{APPENDIX}

The preparation of peptone foams

In the studies described previously in this paper efforts were made to reduce foaming in the inoculated peptone plugs in order to keep the desiccates reasonably compact. As a matter of general interest, however, it was decided to measure 
some recoveries of bacteria from inoculated peptone plugs which had been entirely transformed into foam and dried.

It was found that the plugs could be completely 'collapsed' and dissolved by a very small volume of water or aqueous solution, thus producing a viscous sludge in the bottom of the ampoule. When such a preparation was exposed to high vacuum the sludge expanded rapidly into a foam which filled the greater part of the ampoule (see Pl. 16). The production of the foam was shown to depend upon the minute air bubbles which were trapped in the viscous peptone when the plug was collapsed, the bubbles being originally present as air spaces in the freeze-dried plug. Attempts to produce foams from viscous solutions of peptone prepared from the granular product of the manufacturer met with failure, as also did attempts to produce them from collapsed plugs which had been gently warmed to drive off the trapped air bubbles. Under both circumstances, the viscous material merely spluttered in high vacuum and then dried out gradually from a thick film.

The foams from the plugs solidified extremely rapidly in vacuo. A useful check on water loss could be made by admitting air to the manifold. The foam, if still 'wet', collapsed immediately; if 'dry', it remained intact. The dried foams, despite their fragile appearance, are reasonably resistant to jarring.

The following paragraph describes an experiment in which the recoveries of Vibrio cholerae (624) were measured after a suspension had been dried as a foam. The apparatus and general methods have been given previously in this paper.

Peptone-starch plugs (peptone, $6 \%$; starch, $0.5 \%$ ) were collapsed with $c .0 .08 \mathrm{ml}$. (4 drops) of $10 \%$ glucose. The resultant sludge was inoculated with one drop of a light suspension of the test organism in $20 \%$ glucose. Each sludge, immediately after inoculation, was exposed to high vacuum and $\mathrm{P}_{2} \mathrm{O}_{5}$. Counts were made on the undried suspension and on three dried foams after drying for $24 \mathrm{hr}$. The recoveries were 64,71 and $80 \%$ and were typical of a number of similar such experiments.

In the experiment described the total volume of fluid added to the plug (c. $0.1 \mathrm{ml}$.) was sufficient to produce a foam which filled but did not overflow from the ampoule. These factors had been investigated in preliminary studies.

The composition of the foam may be changed by modifying either the freezedried plug or the solution used to collapse the plug or both. Also, as the solution may be charged with bacteria, the volume of the bacterial inoculum can be increased beyond that used in the experiment described.

Although the usefulness of peptone foams for preserving micro-organisms is not yet known, it has seemed worth while drawing attention to the preparation of them and to emphasize them as structures with enormous surface area yet containable within conveniently small volumes.

I am indebted to the Medical Research Council for a personal research grant. 


\section{REFERENCES}

AnNear, D. I. (1954). Preservation of bacteria. Nature, Lond., 174, 359.

Annear, D. I. (1956). Freeze-drying. Part III. The preservation of micro-organisms. Lab. Practice, 5, 102.

Collier, L. H. (1955). The development of a stable smallpox vaccine. J. Hyg., Camb., 53, 76.

Dubos, R. J. (1949). The Bacterial Cell. Harvard University Press.

Frobisher, M., Parsons, E. I., Pai, S. E. \& Hakim, S. (1947). A simplified method for the preservation of bacteria by desiccation in vacuo. J. Lab. clin. Med. 32, 1008.

Fry, R. M. (1954). The preservation of bacteria. In Biological Application of Freezing and Drying, edited by R. J. C. Harris. Now York: Academic Press Inc.

Fry, R. M. \& Greaves, R. I. N. (1951). The survival of bacteria during and after drying. J. Hyg., Camb., 49, 220.

Greaves, R.I. N. (1954). Theoretical aspects of drying by vacuum sublimation. In Biological Applications of Freezing and Drying, edited by R. J. C. Harris. New York: Academic Press Inc.

Gremf, D. \& Pinkerton, H. (1954). The effects on biological materials of freezing and drying by vacuum sublimation. I. Development and testing of the apparatus. J. exp. Med. $100,81$.

Hegarty, C. P. \& WeEks, O. B. (1940). Sensitivity of Escherichia coli to cold shock during the logarithmic growth phase. J. Bact. 39, 475 .

Heller, G. (1941). A quantitative study of the environmental factors involved in survival and death of bacteria in the desiccated state. J. Bact. 41, 109.

Hutton, R. S., Hilmoe, R. J. \& Roberts, J. L. (1951). Some physical factors that influence the survival of Brucella abortus during freeze-drying. J. Bact. 61, 309.

MrLes, A. A. \& MisRa, S. S. (1938). Estimation of the bactericidal power of blood. J. Hyg., $C a m b ., 38,732$.

NaYlor, H. B. \& Smith, P. A. (1946). Factors affecting the viability of Serratia marcescens during dehydration and storage. J. Bact. 52, 565 .

Proom, H. \& Hemmons, L. M. (1949). The drying and preservation of bacterial cultures. J. gen. Microbiol. 3, 7.

RECORD, B. R. \& TAYLOR, R. (1953). Some factors influencing the survival of Bacterium coli on freeze-drying. J. gen. Microbiol. 9, 475.

SNYDER, T. L. (1947). The relative errors of bacteriological plate-counting methods. J. Bact. $54,641$.

Stamp, LoRd (1947). The preservation of bacteria by drying. J. gen. Microbiol. 1, 251.

\section{EXPLANATION OF PLATES}

Plate 15

Fig. 1. Drying assembly showing Pirani gauge, vacuum pump and manifold. For further explanation see p. 489.

Fig. 2. Uninoculated (A) and inoculated and dried $(B-G)$ freeze-dried plugs. For further explanation see p. 490.

Plate 16

A, uninoculated peptone plug. B, 'collapsed' plug or sludge. C, peptone foam. For further explanation see p. 506 .

(MS. received for publication 8. v. 56) 